\title{
ON A NON-SELF ADJOINT EIGENFUNCTION EXPANSION
}

\author{
D. NAYLOR \\ Department of Applied Mathematics \\ The University of Western Ontario \\ London, Ontario, Canada N6A 5B9
}

(Received June 6, 1983)

ABSTRACT. This paper develops a formula of inversion for an integral transform similar to that associated with the names of Kontorovich and Lebedev. The kernel involves the Hankel function $\mathrm{H}_{\mathrm{u}}^{(1)}(\mathrm{kr})$, in which $r$ varies over a truncated infinite interval $\mathrm{a} \leqq \mathrm{r}<\infty$, where $\mathrm{a}>0$, and the parameter $\mathrm{k}$ is complex. This kind of transform is useful in the investigation of functions that satisfy the Helmholtz equation and the condition of radiation.

KEY WORDS AND PHRASES. Integral transforms, Eigenfunction expansion.', Bes:el fun.tions, Hankel function.

1980 MATHEMATICS SUBJECT CLASSIFICATION CODE. $44 A 15,42 C 10$, SAB:t,

\section{INTRODUCTION.}

In a previous paper, Naylor et al [1], the author investigated the property of an integral transform that can be used in connection with boundary problems for the Helmholtz equation

$$
r^{2} w_{r r}+r w_{r}+k^{2} r^{2} w+w_{\phi \phi}=0
$$

where $(r, \phi)$ are polar coordinates and $r \geqq a>0$. The basic transform in question is that defined by the equation

$$
F(u)=\int_{a}^{\infty} H_{u}^{(1)}(k r) f(r) \frac{d r}{r}
$$

where $\mathrm{H}_{\mathrm{u}}^{(1)}(\mathrm{kr})$ denotes a Hankel function, the notation being that of Watson [2]. The application of the above transform can prove somewhat difficult in that the formal inverse of (1.2) is an eigenfunction expansion that is usually divergent. A valid formula of inversion can be constructed by introducing a suitable convergence factor into the series. In the original investigation it was assumed that the constant $k$ was real and positive, however in some applications this constant is taken to be complex. The introduction of complex values of $k$ into the transform brings into query the validity of inversion formulas initially established only for positive values of $k$. This paper considers the modification necessary to the inversion formula for the transform defined by (1.2) when the constant $k$ is complex. 
It is found that the inverse of (1.2) is still an eigenfunction expansion requiring a summability factor. Since the appearance of such factors in the series renders their application cumbersome, the author, Naylor [3], has proposed alternative transforms such as that defined by the equation

$$
F_{2}(u)=\int_{a}^{\infty} Y_{u}(k r) f(r) \frac{d r}{r}
$$

The inversion formula for this transform involves both a series and an integral each of which is convergent in the classical sense. It should be noted that alternative transforms like (1.3) that are available when $k$ is positive can no longer be employed when $\mathrm{k}$ is complex unless a severe restriction is placed on the asymptotic behaviour of the function $f(r)$ to be expanded. The essential assumption retained in this paper is that $\mathrm{r}^{-1} \mathrm{f}(\mathrm{r}) \in \mathrm{L}(\mathrm{a}, \infty)$. If we write $\mathrm{k}=\mathrm{k}_{1}+i \mathrm{k}_{2}$ where $\mathrm{k}_{1}$, $\mathrm{k}_{2}$ are positive, then $\mathrm{H}_{\mathrm{u}}^{(1)}(\mathrm{kr})$ is $O\left[\mathrm{r}^{-\frac{1}{2}} \exp \left(-\mathrm{k}_{2} \mathrm{r}\right)\right]$ as $\mathrm{r} \rightarrow \infty$, so that the transform defined by (1.2) will exist for functions belonging to the stated class. However $J_{u}(k r)$, $\mathrm{Y}_{\mathrm{u}}(\mathrm{kr})$ and all linear combinations of these functions except $\mathrm{H}_{\mathrm{u}}^{(1)}(\mathrm{kr})$ itself are $0\left[r^{-\frac{1}{2}} \exp \left(k_{2} r\right)\right]$ as $r \rightarrow \infty$, so that (1.3) and similar transforms, apart from $F(u)$, will not in general exist for the class of functions considered in this paper.

The inversion formula for (1.2) constructed in this paper for complex values of $\mathrm{k}$ appears as equation (3.17). In order to establish this formula it is first necessary to obtain a preliminary integral theorem which is proved in the following section of the paper, the proof of the main theorem being given in section 3 .

2. THE INTEGRAL THEOREM.

THEOREM 1. Suppose that $f(r)$ is continuous for $r \geqq a>0$ and that $\mathrm{r}^{-1} \mathrm{f}(\mathrm{r}) \in \mathrm{L}(\mathrm{a}, \infty)$. Let the transform $\mathrm{F}(\mathrm{u})$ be defined by equation (1.2) where $k=k_{1}+i k_{2}$ where $k_{1}>0, k_{2}>0$. Then if $r>a$,

$$
f(r)=\frac{1}{2} \lim _{c \rightarrow 0} \int_{L} u J_{u}(k r) F(u) e^{c u^{2}} d u
$$

where $L$ denotes the imaginary axis of the complex $u$ plane and the parameter $c$ tends to zero through positive values.

To establish the above result a procedure similar to that followed in Naylor [3] is adopted. The expression (1.2) for $\mathrm{F}(\mathrm{u})$ is inserted into the integral present on the right hand side of equation (2.1) and the order of integration is reversed. This procedure leads to the equation

$$
\int_{L} u_{u}(k r) F(u) e^{c u^{2}} d u=\int_{a}^{\infty} f(\rho) \frac{d \rho}{\rho} \int_{L} u J_{u}(k r) H_{u}^{(1)}(k \rho) e^{c u^{2}} d u
$$

In order to justify the inversion of the order of integration it is sufficient to verify that the repeated integral on the right hand side of (2.2) is absolutely convergent. This may be carried out with the aid of the following inequalities, which are established in section 4 of this paper,

$$
\left|J_{\text {is }}(k r)\right| \leftrightharpoons I_{0}\left(k_{2} r\right) \exp \left(\frac{\pi}{2}|s|-s \beta\right)
$$

where $\operatorname{argk}=\beta$ is chosen in the interval $\left(0, \frac{\pi}{2}\right)$, and

$$
\left|H_{\text {is }}^{(1)}(k \rho)\right| \leqq \frac{2}{\pi} K_{0}\left(k_{2} \rho\right) \exp \left(\frac{1}{2} s \pi\right)
$$


It follows from these bounds, which hold on the imaginary axis where $u=$ is and $s$ is real, that the repeated integral in question does not exceed the quantity

$$
\frac{2}{\pi} I_{0}\left(k_{2} r\right) \int_{-\infty}^{\infty} \exp \left[s(\pi-\beta)-c s^{2}\right] \operatorname{sds} \int_{a}^{\infty} \rho^{-1}|f(\rho)| k_{0}\left(k_{2} \rho\right) d \rho
$$

This expression is finite since $\mathrm{K}_{\mathrm{o}}\left(\mathrm{k}_{2} \rho\right)=0\left[\rho^{-\frac{1}{2}} \exp \left(-\mathrm{k}_{2} \rho\right)\right]$ as $\rho \rightarrow \infty$. This estab1ishes the truth of $(2.2)$.

Next it will be demonstrated that

$$
\int_{L} u\left[J_{u}(k r) H_{u}^{(1)}(k \rho)-J_{u}(k \rho) H_{u}^{(1)}(k r)\right] e^{c u^{2}} d u=0
$$

This result is true because the integrand is an odd function of $u$, a result which is easily verified with the aid of the identity

$$
H_{u}^{(1)}(x)=\frac{1}{i \sin u \pi}\left[J_{-u}(x)-e^{-i u^{\top}} J_{u}(x)\right]
$$

from which it follows that

$$
J_{u}(k r) H_{u}^{(1)}(k \rho)-J_{u}(k \rho) H_{u}^{(1)}(k r)=\frac{1}{i \sin u \pi}\left[J_{u}(k r) J_{-u}(k \rho)-J_{u}(k \rho) J_{-u}(k r)\right]
$$

This expression is an even function of $u$, since it is evident that the cross product of Bessel functions on the right hand side of (2.7) is an odd function of $u$, so that (2.5) follows. Hence

$$
\int_{L} u J_{u}(k r) H_{u}^{(1)}(k \rho) e^{c u^{2}} d u=\int_{L} u J_{u}(k \rho) H_{u}^{(1)}(k r) e^{c u^{2}} d u
$$

If the domain of the p-integration occurring in the integral on the right hand side of (2.2) is separated into the parts $\rho \geqq r$ and $D \leqq r$, and the relation (2.8) applied to transform the contribution from the second such part we find that

$$
\begin{gathered}
\int_{L} u J u_{u}(k r) F(u) e^{c u^{2}} d u=\int_{a}^{r} \rho^{-1} f(\rho) d \rho \int_{L} u J(k \rho) H_{u}^{(1)}(k r) e^{c u^{2}} d u \\
+\int_{r}^{\infty} \rho^{-1} f(\rho) d \rho \int_{L} u_{u}(k r) H_{u}^{(1)}(k r) e^{c u^{2}} d u
\end{gathered}
$$

The Bessel functions present in (2.9) are now eliminated in favour of the function $g(u, r, \rho)$ defined by the equation

$$
g(u, r, \rho)=u J_{u}(k r) H_{u}^{(1)}(k \rho)-\frac{1}{i \pi}(r / \rho)^{u}
$$

This leads to the equation

$$
\begin{gathered}
\int_{L} u J_{u}(k r) F(u) e^{c u^{2}} d u=\frac{1}{i \pi} \int_{a}^{r} \rho^{-1} f(\rho) d \rho \int_{L}(\rho / r) e^{c u^{2}} d u \\
\quad+\frac{1}{i \pi} \int_{r}^{\infty} \rho^{-1} f(\rho) d \rho \int_{L}(r / \rho)^{u} e^{c u^{2}} d u+I_{1}+I_{2}
\end{gathered}
$$

where

$$
\begin{aligned}
& I_{1}=\int_{a}^{r} \rho^{-1} f(\rho) d \rho \int_{L} g(u, \rho, r) e^{c u^{2}} d u \\
& I_{2}=\int_{r}^{\infty} \rho^{-1} f(\rho) d \rho \int_{L} g(u, r, \rho) e^{c u^{2}} d u
\end{aligned}
$$

The two L-integrals present on the right hand side of (2.11) are equal to one another, as is evident upon changing the variable of integration from $u$ to $-u$. The cor- 
responding repeated integrals may therefore be combined to yield the equation

$$
\begin{aligned}
\int_{L} u_{u}(k r) F(u) e^{c u^{2}} d u & =\frac{1}{i \pi} \int_{a}^{\infty} \rho^{-1} f(\rho) d \rho \int_{L}(\rho / r) e^{c u^{2}} d u+I_{1}+I_{2} \\
& =\frac{1}{i \pi} \int_{L} r^{-u} e^{c u^{2}} d u \int_{a}^{\infty} \rho^{u-1} f(\rho) d \rho+I_{1}+I_{2}
\end{aligned}
$$

after a further change in the order of integration, which is again possible since $\mathrm{u}=\mathrm{is}$ on the path $\mathrm{L}$ so that the modulus of the integrand is $\rho^{-1}|f(\rho)| e^{-c^{2}}$ which is integrable for any $c>0$. The integral now present on the right hand side of (2.14) reduces when $c=0$ to $2 i \pi f(r)$ by the Mellin inversion theorem, Titchmarsh [4, p. 46], and it is uniformly convergent for $c \geq 0$. Hence, on letting $c \rightarrow 0$ in $(2.14)$, we $f$ ind the equation

$$
\lim _{c \rightarrow 0} \int_{L} u J(k r) F(u) e^{c u^{2}} d u=2 f(r)+\lim _{c \rightarrow 0}\left(I_{1}+I_{2}\right)
$$

It will now be verified that the quantities $I_{1}$ and $I_{2}$ each tend to zero as $c \rightarrow 0$. To do this the contour integrals appearing in the formulas (2.12), (2.13) will be transformed by means of Cauchy's theorem, in which the path $\mathrm{L}$ is deformed onto the path $\mathrm{W}$, depicted in figure 1 . This path consists of the rays argu $= \pm \alpha$ where $\frac{\pi}{4}<\alpha<\frac{\pi}{2}$. In order to carry out this procedure it is first necessary to determine the behaviour of the function $g(u, r, p)$ when $u$ is large and this can be found from equation (2.10) after substituting the known asymptotic forms for the Bessel functions. The behaviour of the J-type Bessel function is obtained from the equation

$$
J_{u}(x)=\frac{(x / 2)^{u}}{\Gamma(u+1)}\left[1-\frac{x^{2}}{4(u+1)}+0\left(u^{-2}\right)\right]
$$

which applies whenever $\mathrm{u}$ is large compared with $\mathrm{x}$ and bounded away from the negative integers. The behaviour of the Hankel functions can be found by inserting (2.16) into the formula (2.6) which leads to the equation

$$
H_{u}^{(1)}(x)=\left[\frac{\Gamma(u)(2 / x)^{u}}{i \pi}-\frac{e^{-i u \pi}(x / 2)^{u}}{i \Gamma(u+1) \sin u \pi}\right]\left[1+0\left(u^{-1}\right)\right]
$$

It also follows from (2.6) and (2.16) that the function $g(u, r, n)$ defined by $(2.10)$ possesses the asymptotic formula

$$
g(u, r, \rho)=-\left[\frac{k^{2}\left(r^{2}-\rho^{2}\right)(r / \rho)^{u}}{4 i u \pi}+\frac{\left(k^{2} r \rho / 4\right)^{u} e^{-i u \pi}}{i \Gamma(u) \Gamma(u+1) \sin u \pi}\right]\left[1+0\left(u^{-1}\right)\right]
$$

The asymptotic behaviour of the $\mathrm{T}$-functions appearing in these formulas can be obtained from Stirling's formula

$$
\Gamma(u)=(2 \pi / u)^{\frac{1}{2}} \exp (u \log u-u)\left[1+0\left(u^{-1}\right)\right]
$$

which applies as $\mathrm{u} \rightarrow \infty$ in $\mid$ argu $\mid \leqq \pi-\delta$, and which when substituted into (2.18) leads to the equation

$$
g(u, r, \rho)=-\left[\frac{k^{2}\left(r^{2}-\rho^{2}\right)(r / \rho)^{u}}{4 i u \pi}+\frac{\left(k^{2} r \rho / 4\right)^{u} \exp (-2 u \log u+2 u-i u)}{2 i \pi \sin u \pi}\right]\left[1+0\left(u^{-1}\right)\right]
$$

This equation applies for sufficiently large values of $u$ bounded away from the integers and it holds uniformly in any bounded domain of values of $\rho$. If we set 
$u=\operatorname{Re}^{i \theta}$ in the second term of (2.20) we find the formula

$$
\begin{gathered}
g(u, r, \rho)=-\left[\frac{k^{2}\left(r^{2}-\rho^{2}\right)(r / \rho)^{u}}{4 i u \pi}+0\left\{\frac { ( k ^ { 2 } r \rho / 4 ) ^ { u } } { \operatorname { s i n } u \pi } \operatorname { e x p } \left\{-2 R \cos \theta \log \left(\frac{R}{e}\right)\right.\right.\right. \\
+2 R(\theta+\pi) \sin \theta\}\}]\left[1+0\left(u^{-1}\right)\right]
\end{gathered}
$$

With the aid of this expression it is possible to justify the proposed deformation of the path of integration. Since the Bessel functions are entire functions of the complex variable $u$ the same is true of the function $g(u, r, \rho)$. When $R$ is large the dominant term present in the exponential in (2.21) is the logarithmic one, except in the vicinity of the imaginary axis where $\theta$ is close or equal to $\frac{ \pm \pi}{2}$. However the modulus of the summability factor is $\exp \left(c \mathrm{R}^{2} \cos 2 \theta\right)$ and $\cos 2 \theta$ is negative in the region traversed. This factor tends to zero sufficiently rapidly as $R \rightarrow \infty$ to permit the path $L$ to be deformed onto $W$ and this leads to the equation

$$
\int_{L} g(u, r, \rho) e^{c u^{2}} d u=\int_{W} g(u, r, \rho) e^{c u^{2}} d u
$$

Now it follows from (2.21) that the function $g(u, r, i)$ is absolutely integrable on $W$ for values of $\rho$ such that $\rho>r$ so that, on taking the limit of each side of the preceding equation as $c \rightarrow 0$,

$$
\lim _{c \rightarrow 0} \int_{L} g(u, r, \rho) e^{c u^{2}} d u=\int_{W} g(u, r, \rho) d u
$$

The integral appearing on the right hand side of this equation may be evaluated by closing the contour on the right hand side of $W$ by means of a sequence of circular arcs of radii $|u|=n+\frac{1}{2}$, where $n \rightarrow \infty$, and applying Cauchy's theorem. Since $g=0\left[u^{-1}(r / \rho)^{u}\right]$ the contribution from the circular arc is zero in the 1 imit as $\mathrm{n} \rightarrow \infty$ provided that $\rho>\mathrm{r}$. For such values of $\rho$ it follows that

$$
\lim _{c \rightarrow 0} \int_{L} g(u, r, \rho) e^{c u^{2}} d u=0
$$

This result will first be applied to prove that the limiting value of $I_{1}$ as $c \rightarrow 0$ is zero. On interchanging $r, \rho$ in (2.23) we find that

$$
\lim _{c \rightarrow 0} \int_{L} g(u, \rho, r) e^{c u^{2}} d u=0
$$

whenever $\rho<\mathbf{r}$. Since the convergence is uniform for $a \leqq \rho \leqq r-\varepsilon$ it follows that

$$
\lim _{c \rightarrow 0} \int_{a}^{r-\varepsilon} f(\rho) \frac{d \rho}{\rho} \int_{L}^{\rho} g(u, \rho, r) e^{c u^{2}} d u=0
$$

To complete the verification that $\operatorname{lim~} I_{1}=0$ it remains, in view of the preceding result and the definition (2.12), to prove that

$$
\lim _{c \rightarrow 0} \int_{r-\varepsilon}^{r} f(\rho) \frac{d \rho}{\rho} \int_{L} g(u, r, r) e^{c u^{2}} d u=0
$$

Since $\varepsilon$ is arbitrarily small it is sufficient to verify that the L-integral in (2.24) is bounded uniformly for all relevant values of $\rho$ and $c$. This is carried out by deforming $L$ onto $W$ which is then decomposed into a distant part $W_{1}$, on which the estimate (2.21) applies, together with the remaining (bounded) part $\mathrm{W}_{2}$. This leads to a consideration of the integral 


$$
\int_{W_{1}} g(u, \rho, r) e^{c u^{2}} d u
$$

Since $u=R e^{ \pm i x}$ on $W_{1}$ it is seen that the contribution to the above integral of the leading term in the expression (2.21) for $g$ has a modulus that does not exceed the quantity

$$
\frac{k^{2}\left(r^{2}-\rho^{2}\right)}{4 \pi} \int_{R_{1}}^{\infty} R^{-1}(\rho / r)^{R \cos \alpha} d R
$$

Since $\rho / r \leqq \exp [(\rho / r)-1]$ for $\rho \leqq r$ the above expression is less than

$$
\begin{aligned}
& \frac{k^{2}\left(r^{2}-\rho^{2}\right)}{4 \pi R_{1}} \int_{R_{1}}^{\infty} \exp \left[-\left(1-\frac{\rho}{r}\right) R \cos \alpha\right] d R \\
& =\frac{r^{2}(r+\rho)}{4 \pi R_{1} \cos \alpha} \exp \left[-\left(1-\frac{\rho}{r}\right) R_{1} \cos \alpha\right] \\
& \leqq \frac{2 k^{2} r^{2}}{4 \pi R_{1} \cos \alpha}
\end{aligned}
$$

The contribution of the finite part $W_{2}$ is clearly bounded uniformly for $c \geqq 0$, as is that from the remaining term in (2.21) so that (2.24) follows.

To prove that $\operatorname{lim~} \mathrm{I}_{2}=0$ we again deform $\mathrm{L}$ onto $\mathrm{W}$ and then select $r_{1}$ in accordance with (4.5) and $r_{0}$ greater than $r_{1}$ and large enough to ensure that

$$
\int_{\mathrm{r}}^{\infty}|\mathrm{f}(\rho)| \frac{\mathrm{d} \rho}{\rho} \int_{W}|\mathrm{~g}(\mathrm{u}, \mathrm{r}, \rho) \mathrm{du}|<\varepsilon
$$

This choice is possible since $\rho^{-1} f(\rho) \varepsilon L(a, \infty)$ whilst, as the following argument shows, the W-integral exists. If we appeal to the formulas (2.16) and the bound (4.3) we find, after using Stirling's formula, that, for $\rho \geqq r_{1}$ and $u$ on $W$, $\mathrm{uJ}_{\mathrm{u}}(\mathrm{kr}) \mathrm{H}_{\mathrm{u}}^{(1)}(\mathrm{k} \rho)=0\left[\exp \left\{-\mathrm{R} \cos \alpha \log \left|\frac{\mathrm{k}_{2} \mathrm{r}_{1}}{\mathrm{kr} \cos \alpha}\right|+\pi \mathrm{R} \sin \alpha\right\}\right]$

Since $r_{1}$ is chosen to satisfy (4.5), the above expression is integrable along $W$. The remaining term appearing in the definition of $g$ is also absolutely integrable along $W$ since $\rho \geqq r_{1}>r$ so that the same is true of $g$ itself. Arguments identical with those already used in the treatment of $I_{1}$ show that the contributions of the remaining intervals $(r, r+\varepsilon)$ and $\left(r+\varepsilon, r_{0}\right)$ vanish in the limit as $\mathrm{c} \rightarrow 0$.

3. THE EXPANSION THEOREM.

To obtain the explicit eigenfunction expansion the formula (2.1) will be combined with the following identity:

$$
\begin{aligned}
\frac{1}{2} \lim _{c \rightarrow 0} \int_{L} \frac{u u^{(k a) H_{u}^{(1)}(k r) F(u) e^{c u^{2}} d u}}{H_{u}^{(1)}(k a)} \\
\quad=-i \pi \lim _{c \rightarrow 0} \sum_{u_{n}} \frac{u_{u}(k a) H_{u}^{(1)}(k r) F(u) e^{c u^{2}}}{(\partial / \partial u) H_{u}^{(1)}(k a)}
\end{aligned}
$$

in which the summation in the series on the right hand side of this equation extends over all of those zeros $u_{n}$ of the function $\mathrm{H}_{u}^{(1)}(k a)$ that are positioned in the half plane $\operatorname{Re}(u)>0$. 
Before proceeding to establish the above formula it is first necessary to show that the zeros $u_{n}$ of sufficiently large magnitude are confined to the sectors $\frac{\pi}{2}-\delta<\operatorname{argu}_{n}<\frac{\pi}{2}$ and $\frac{-\pi}{2}-\delta<\operatorname{argu}_{n}<\frac{-\pi}{2}$, where $\delta$ is a small positive angle. The zeros of the Hankel function regarded as a function of its order are discussed in Cochran [5], Keller et al [6], and Magnus \& Kotin [7]. It can be shown by a classical type argument, based on the differential equation satisfied by the Hankel function, similar to that followed in Naylor [8], where the zeros of the function $\mathrm{K}_{\mathrm{u}}(\mathrm{x})$ for fixed positive $x$ are discussed, that the zeros $u=t+$ is of $H_{u}^{(1)}(k a)$, where $k=k_{1}+i k_{2}$, satisfy the relation $s t \geqq a^{2} k_{1} k_{2}$. Since attention in this paper is confined to positive values of $k_{1}$ and $k_{2}$ it follows that $s t>0$ and that the zeros in question are located in the first and third quadrants of the complex u-plane. There are no real and no purely imaginary zeros. Since $H_{-u}^{(1)}(k a)=e{ }^{i u \pi}{ }_{u}^{(1)}(k a)$ it also follows that to each zero $u_{n}$ in the first quadrant there corresponds a zero $-u_{n}$ in the third quadrant of the complex u-plane. Only the zeros of the first quadrant are pertinent to the expansion formula developed in this paper.

To establish the location of the large zeros in the stated sectors it is necessary to estimate their values by means of equation (2.17) in which the $\Gamma$-functions are themselves estimated by means of Stirling's formula (2.19). We find, after some reduction, that, for $\operatorname{Im}(u)>0$,

$$
H_{u}^{(1)}(k a)=-2 e^{\frac{1}{4 i \pi}}(2 / \pi u)^{\frac{1}{2}} \sinh \left[u \log (2 u / k a e)+\frac{1}{4} i \pi\right]\left[1+0\left(u^{-1}\right)\right]
$$

If we set $u=\operatorname{Re} i \theta, k=p e^{i \beta}$ where $R>0, p>0$, and $0<\beta<\frac{\pi}{2}$ we find that

$$
H_{u}^{(1)}(k a)=-2 e^{\frac{1}{4} i \pi}(2 / \pi u)^{\frac{1}{2}} \sinh (\phi+i \psi)\left[1+0\left(R^{-1)}\right]\right.
$$

where

$$
\begin{aligned}
& \phi=R \cos \theta \log (2 R / \text { pae })-R(\theta-\beta) \sin \theta \\
& \psi=R \sin \left(\log (2 R / \text { pae })+R(\theta-\beta) \cos \theta+\frac{\pi}{4}\right.
\end{aligned}
$$

The large zeros occur at the points $u_{n}=R_{n} e^{i \theta n}$ determined by the equations $\phi=0$, $\psi=\mathrm{n} \pi$. On solving these equations we find the approximate solution

where

$$
\theta_{n}=\frac{\pi}{2}-\frac{(\pi-2 \beta)}{2 \log \left(2 R_{n} / p a\right)}
$$

$$
R_{n} \log \left(2 R_{n} / p a e\right)=\left(n-\frac{1}{4}\right)_{\pi}
$$

and $n$ is a large positive integer. It is seen from (3.6) that $\theta_{n} \rightarrow \frac{\pi}{2}$ as $n$ and hence $R_{n}$ tend to infinity, so that the zeros of sufficiently large modulus are 1ocated inside some sector $\frac{\pi}{2}-\delta \leqq \theta<\frac{\pi}{2}$ where $\delta$ is a small positive angle.

The existence of the integral appearing in (3.1) can be inferred from the following asymptotic formulas which hold on the positive imaginary axis, where $u=$ is and $s>0$,

$$
\begin{aligned}
\mathrm{J}_{\text {is }}(\mathrm{x}) & =(2 \pi s)^{-\frac{1}{2}} \exp \left[-\mathrm{is} \log (2 \mathrm{~s} / \mathrm{xe})+\frac{1}{2} \mathrm{~s} \pi-\frac{1}{4} \mathrm{i} \pi\right]\left[1+0\left(\mathrm{~s}^{-1}\right)\right] \\
\mathrm{H}_{\text {is }}^{(1)}(\mathrm{x}) & =(2 / \pi s)^{-\frac{1}{2}} \exp \left[-\mathrm{is} \log (2 \mathrm{~s} / \mathrm{xe})+\frac{1}{2} \mathrm{~s} \pi-\frac{1}{4} \mathrm{i} \pi\right]\left[1+0\left(\mathrm{~s}^{-1}\right)\right] \\
\mathrm{F}(\mathrm{is}) & =0\left[\exp \left|\frac{1}{2} \mathrm{~s} \pi\right|\right]
\end{aligned}
$$


The above formulas follow from (2.16), (2.17) after utilizing (2.19), whilst the expression for $F($ is) is established in section 4 of this paper where it appears as equation (4.9). The formula giving the behaviour of the Hankel function when $s$ is large and negative is obtained by making use of the identity $H_{-i s}^{(1)}(x)=e^{-s \pi} H_{i s}^{(1)}(x)$. The corresponding formula for $J_{i s}(x)$ for $s$ large and negative is given by the equation

$$
J_{-i s}(x)=(2 \pi s)^{-\frac{1}{2}} \exp \left[\text { is } \log (2 s / x e)+\frac{1}{2} \pi s+\frac{1}{4} i n\right]\left[1+0\left(s^{-1}\right)\right]
$$

which applies for $s \rightarrow+\infty$.

On combining these expressions it is found that the integrand occurring in (3.1) is $0\left[s^{\frac{1}{2}} \exp \left(-c s^{2}-|\pi s|+s \beta\right)\right]$ as $|s| \rightarrow \infty$ so that the integral is convergent provided that $c>0$.

To prove (3.1) the path of integration $L$ is first deformed onto the path $W_{0}$ illustrated in figure 2. This path consists of (i) the segment of the line $\operatorname{Re}(u)=$ intercepted between the rays argu $= \pm \alpha$ and (ii) the parts of these rays that lie to the right of this line. The quantity $\lambda$ is chosen large enough to ensure that all of the zeros $u_{n}$ lie to the left of $w_{o}$, and the angle $x$ is defined as in $\bar{s}$ to lie in the interval $\left(\frac{\pi}{4}, \frac{\pi}{2}\right)$.

To justify the proposed deformation the paths $L$ and $W_{O}$ are connected by a sequence of curves $C_{n}$ which recede to infinity and which avoid the zeros $u_{n}$. Since these zeros lie on the curve for which $\psi=n \pi$ we may choose the curve $C_{n}$ to be that whose polar equation in the sector $\frac{\pi}{2}-\delta \leqq \theta \leqq \frac{\pi}{2}$ is given by the equation $\psi=\left(n+\frac{1}{2}\right) \pi$ and which is continued beyond this sector by means of a circular arc of suitable radius $\rho_{n}$ to meet the ray argu $=\alpha$. The part of $C_{n}$ located in the sector $\frac{-\pi}{2} \leqq \theta \leq-\alpha$ may also be taken to be an arc of a circle of radius $p_{n}$. To estimate the behaviour of the Hankel functions on the paths $C_{n}$ we replace $a$ by $r$ in (3.2) which, in view of the definitions of $\phi$ and $\psi$, leads to the equations

$$
\mathrm{H}_{\mathrm{u}}^{(1)}(\mathrm{kr})=-2 \mathrm{e}^{\frac{1}{4} \mathrm{i} \pi}(2 / \pi \mathrm{u})^{\frac{1}{2}} \sinh [\phi+i \psi+\mathrm{u} \log (\mathrm{a} / \mathrm{r})]\left[1+0\left(\mathrm{u}^{-1}\right)\right]
$$

On the parts of $C_{n}$ lying inside the sector where $\psi=\left(n+\frac{1}{2}\right) \pi$ the above equation reduces to

$$
H_{u}^{(1)}(k r)=-2 i e^{\frac{1}{4} i \pi}(2 / \pi u)^{\frac{1}{2}} \cos n \pi \cosh [\phi+u \log (a / r)]\left[1+0\left(u^{-1}\right)\right]
$$

so that

$$
H_{u}^{(1)}(k a)=-2 i e^{\frac{1}{4} i \pi}(2 / \pi u)^{\frac{1}{2}} \cos n \pi \cosh \phi\left[1+0\left(u^{-1}\right)\right]
$$

and therefore

$$
\begin{aligned}
\left|\frac{\mathrm{H}_{\mathrm{u}}^{(1)}(\mathrm{kr})}{\mathrm{H}_{\mathrm{u}}^{(1)}(\mathrm{ka})}\right| & \sim \frac{\cosh [\phi+\mathrm{u} \log (\mathrm{a} / \mathrm{r})]}{\cosh \phi} \\
& \leqq \frac{\cosh [\phi+\mathrm{R} \cos \theta \log (\mathrm{a} / \mathrm{r})]}{\cosh \phi} \\
& \leqq \cosh [R \cos \theta \log (\mathrm{a} / \mathrm{r})]+\tanh \phi \sinh \left[R \cos \theta \log \left(\frac{\mathrm{a}}{\mathrm{r}}\right)\right] \\
& \leqq \exp [R \cos \theta \log (\mathrm{r} / \mathrm{a})]
\end{aligned}
$$

The behaviour of the Hankel function outside the sector $\frac{\pi}{2}-\delta \cdot \theta \leqq \frac{1}{2}$ is obtained 
from (2.17) and (2.19). This leads to the equation

$$
\mathrm{H}_{\mathrm{u}}^{(1)}(\mathrm{kr})=\frac{\Gamma(\mathrm{u})}{\mathrm{i} \pi}(2 / \mathrm{kr}) \mathrm{u}\left[1-\frac{\mathrm{e}^{-\mathrm{iu} \pi-2 \mathrm{u} \log (2 \mathrm{u} / \mathrm{ker})}}{2 \sin \mathrm{u} \pi}\right]\left[1+0\left(\mathrm{u}^{-1}\right)\right]
$$

Since $|\sin u \pi| \sim \frac{1}{2} \exp |\pi R \sin \theta|$ whenever $\mid$ Imu| is large it follows that, on $C_{n}$, $\mathrm{H}_{\mathrm{u}}^{(1)}(\mathrm{kr})=\frac{\Gamma(\mathrm{u})}{\mathrm{i} \pi}\left(\frac{2}{\mathrm{kr}}\right)^{\mathrm{u}}\left[1+0\left(\exp \left\{-2 \mathrm{R} \cos \theta \log \left|\frac{2 \mathrm{R}}{\mathrm{ker}}\right|+R \sin \theta(\pi+2 \theta-2 \beta)-|\pi \operatorname{Rs} \operatorname{in} \theta|\right\}\right)\right]$

The 0-term in this formula tends to zero as $R \rightarrow \infty$ for all values of $\theta$ in the relevant intervals $\frac{-\pi}{2} \leqq \theta \leqq-\alpha$ and $\alpha \leqq \theta \leqq \frac{\pi}{2}-\delta$, the dominant term in the exponential being the logarithmic one. Therefore

$$
\frac{\mathrm{H}_{\mathrm{u}}^{(1)}(\mathrm{kr})}{\mathrm{u}^{(1)}(\mathrm{ka})} \sim\left(\frac{\mathrm{a}}{\mathrm{r}}\right)^{\mathrm{u}}
$$

for large $u$ in the sectors $\frac{-\pi}{2} \leqq$ argu $\leqq-\alpha$ and $\alpha \leqq$ argu $\leqq \frac{\pi}{2}-\delta$. This formula also applies in the larger interval $\frac{-\pi}{2} \leqq$ argu $\leqq \frac{\pi}{2}-\delta$ provided that $u$ is bounded away from the large zeros of sinum. We can ensure this by choosing $\operatorname{Re}(u)=n+\frac{1}{2}$ whenever $u$ is in the sector $-\delta \leqq$ argu $\leqq \delta$ so that $|\operatorname{sinu} \pi|=\cosh |\pi \operatorname{Rin} \theta| \operatorname{therein}$. With this restriction we see that (3.8) holds for large $u$ throughout the sector $\frac{-\pi}{2} \leqq$ argu $\leqq \frac{\pi}{2}-\delta$.

On combining (2.16) and (4.8) with (3.7) or (3.8), as the case may be, we find that

$$
\frac{\mathrm{uJ}_{\mathrm{u}}(\mathrm{ka}) \mathrm{H}_{\mathrm{u}}^{(1)}(\mathrm{kr}) \mathrm{F}(\mathrm{u})}{\mathrm{H}_{\mathrm{u}}^{(1)}(\mathrm{ka})}=0\left[\left(\frac{\mathrm{r}}{\mathrm{a}}\right)^{\mathrm{u}}\right]+0\left[\frac{\mathrm{e}^{-\mathrm{iu \pi}}\left(\mathrm{k}^{2} \mathrm{rr} / \mathrm{l}\right)^{\mathrm{u}}}{\Gamma(\mathrm{u}) \Gamma(\mathrm{u}+1) \mathrm{sinu} \pi}\right]+0\left[\frac{\mathrm{e}^{\frac{1}{2} \mathrm{~s} \pi} \Gamma(\mathrm{t})\left(\mathrm{kr} / \mathrm{k}_{2} \mathrm{r}_{1}\right)^{\mathrm{u}}}{\Gamma(\mathrm{u})}\right]
$$

for $\frac{\pi}{2}-\delta \leqq \theta \leqq \frac{\pi}{2}$, and

$$
\frac{\mathrm{uJ}(\mathrm{u} a) \mathrm{H}_{\mathrm{u}}^{(\mathrm{l})}(\mathrm{kr}) \mathrm{F}(\mathrm{u})}{\mathrm{H}_{\mathrm{u}}^{(1)}(\mathrm{ka})}=0\left[\left(\frac{\mathrm{a}}{\mathrm{r}}\right)^{\mathrm{u}}\right]+0\left[\frac{\mathrm{e}^{-\mathrm{iu \pi}\left(\mathrm{k}^{2} \mathrm{a}^{2} \mathrm{r}_{1} / 4 \mathrm{r}\right)^{\mathrm{u}}}}{\Gamma(\mathrm{u}) \Gamma(\mathrm{u}+1) \sin \mathrm{u}^{\pi}}\right]+0\left[\frac{\mathrm{e}^{\frac{1}{2} \mathrm{~s} \pi} \Gamma(\mathrm{t})\left(\mathrm{ka}^{2} / \mathrm{k}_{2} \mathrm{rr}\right)^{\mathrm{u}}}{\Gamma(\mathrm{u})}\right]
$$

for $\frac{-\pi}{2} \leqq \theta \leqq \frac{\pi}{2}-\delta$. The quantity $r_{1}$ appearing in the above formulas is chosen in accordance with the inequality (4.5). Since the function $\Gamma(t)$ is unbounded as $t \rightarrow 0$, i.e. as $\theta \rightarrow \frac{ \pm \pi}{2}$ the formulas (3.9), (3.10) cannot be applied in this vicinity where however we may use the simpler estimate

$$
\frac{\mathrm{uJ}_{\mathrm{u}}(\mathrm{ka}) \mathrm{H}_{\mathrm{u}}^{(\mathrm{l})}(\mathrm{kr}) \mathrm{F}(\mathrm{u})}{\mathrm{H}_{\mathrm{u}}^{(1)}(\mathrm{ka})}=0\left[\frac{(\mathrm{kr} / 2) \mathrm{e}^{\frac{1}{2} \mathrm{~s} \pi}}{\Gamma(\mathrm{u}+1)}\right]
$$

This bound, which follows from (2.16), (3.7) and (4.9) applies as $u \rightarrow \infty$ in the strip $0 \leqq \operatorname{Re}(u) \leqq \lambda$. In justifying the deformation of $L$ onto $W_{0}$, it is necessary to carry out the deformation in two stages, in which the path $\mathrm{L}$ is first moved onto the line $\operatorname{Re}(\mathrm{u})=\lambda$. The bound on the right hand side of (3.11) is $0\left(e^{s \pi}\right)$ as $s \rightarrow+\infty$ and $O(1)$ as $s \rightarrow-\infty$ but the presence of the summability factor, which has modulus of $0\left[\exp \left(-c s^{2}\right)\right]$ in the region traversed, ensures that the integrand in (3.1) tends to zero as $s \rightarrow \pm \infty$, provided that $c>0$. The bounds (3.9) and (3.10) may then be used in justifying the deformation of the path from the 1 ine $\operatorname{Re}(u)=1$ onto $W_{0}$. 
The behaviour of the $\Gamma$-functions in these bounds is given by Stirling's formula which shows that

$$
\begin{aligned}
|\Gamma(u)| & =(2 \pi / R)^{\frac{1}{2}} \exp [R \cos \theta \log (R / e)-R \theta \sin \theta]\left[1+O\left(R^{-1}\right)\right] \\
\Gamma(t) & =(2 \pi / t)^{\frac{1}{2}} \exp [t \log (t / e)]\left[1+0\left(t^{-1}\right)\right]
\end{aligned}
$$

An inspection of the above expressions reveals that the presence of the summability factor, which $0\left[\exp \left(c R^{2} \cos 2 \theta\right)\right]$ where $\cos 2 \theta<0$ in the region traversed, ensures that the integrand in (3.1) tends to zero sufficiently rapidly as the path $\mathrm{C}_{n}$ recedes to infinity to permit the contour $L$ to be deformed onto $W_{0}$ as described.

On evaluating the residues at the poles of the integrand we find the equation

$$
\begin{aligned}
& \int_{L} \frac{u_{u}(k a) H_{u}^{(1)}(k r) F(u) e^{c u^{2}} d u}{H_{u}^{(1)}(k a)}=\int_{W_{O}} \frac{u_{u}(k a) H_{u}^{(1)}(k r) F(u) e^{c u^{2}} d u}{H_{u}^{(1)}(k a)} \\
& +2 i \pi \sum \frac{u_{u}(k a) H_{u}^{(1)}(k r) F(u) e^{c u^{2}}}{(\partial / \partial u) H_{u}^{(1)}(k a)}
\end{aligned}
$$

in which the summation extends over all of the zeros $u_{n}$ that are positioned in the half plane $\operatorname{Re}(u)>0$.

The final step necessary to establish the validity of (3.1) is to prove that

$$
\lim _{c \rightarrow 0} \int_{W_{0}} \frac{u_{u}(k a) H_{u}^{(1)}(k r) F(u) e^{c u^{2}} d u}{H_{u}^{(1)}(k a)}=\int_{W_{0}} \frac{u J_{u}(k a) H_{u}^{(1)}(k r) F(u) d u}{H_{u}^{(1)}(k a)}=0
$$

With this aim in view, it will first be proved that the integral on the right hand side of (3.15) is absolutely convergent. This will imply, since $\left|\exp \left(\mathrm{cu}^{2}\right)\right| \leqq 1$ on $w_{o}$, that the integral on the left hand side of (3.15) is uniformly convergent for $c \geqq 0$ so that the value of the limit as $c \rightarrow 0$ may be obtained by setting $c=0$ in the integrand. The integral on the right hand side of (3.15) can then easily be shown to be equal to zero by closing the contour on the right hand side of $W_{O}$ and applying Cauchy's theorem.

To verify these results we first note that the asymptotic form of the integrand in (3.15) is given immediately by equation (3.10) after substituting the expressions (3.12) and (3.13) for the $\Gamma$-functions. Since $|\sin u \pi| \sim \frac{1}{2} \exp |R \sin \theta|$ as $u \rightarrow \infty$ along any ray $u=\operatorname{Re}^{i \theta}$ for which $\theta \neq 0$ we find that

$$
\begin{aligned}
\frac{\mathrm{uJ}_{\mathrm{u}}(\mathrm{ka}) \mathrm{H}_{\mathrm{u}}^{(\mathrm{l})}(\mathrm{kr}) \mathrm{F}(\mathrm{u})}{\mathrm{H}_{\mathrm{u}}^{(1)}(\mathrm{ka})}= & 0\{\exp [-\mathrm{R} \cos \theta \log (\mathrm{r} / \mathrm{a})]\}+0\{\exp [-2 \mathrm{R} \cos \theta \log (\mathrm{R} / \mathrm{e}) \\
& \left.\left.+\mathrm{R} \cos \theta \log \left|\mathrm{k}^{2} \mathrm{a}^{2} \mathrm{r}_{1} / 4 \mathrm{r}\right|+\mathrm{R} \sin \theta(\pi+2 \theta-2 \beta)-\pi|\mathrm{R} \sin \theta|\right]\right\} \\
& +0\left\{\exp \left[\mathrm{R} \cos \theta \log \left|\mathrm{ka}^{2} \cos \theta / \mathrm{k}_{2} \mathrm{rr}_{1}\right|+\mathrm{R}\left(\theta+\frac{\pi}{2}-\beta\right) \sin \theta\right]\right\}
\end{aligned}
$$

The behaviour of the integrand on $W_{0}$ is obtained by setting $\theta= \pm \alpha$ in the above expression. Since $r>a$ the first term on the right hand side of the preceding expression is absolutely integrable on $W_{0}$. The same conclusion applies to the second term, the dominant term therein being that involving $\mathrm{R} \log \mathrm{R}$. With regard to the 
third 0 -term in (3.16) we note that, since $|\theta| \leqq \frac{\pi}{2}, B>0$ and $r>a$, the argument of the exponential function in this term cannot exceed the quantity

$$
\begin{aligned}
& -\mathrm{R} \cos \theta \log \left|\mathrm{k}_{2} \mathrm{rr}_{1} /\left(\mathrm{ka}^{2}\right)\right|+\pi \mathrm{R} \sin \theta \\
\leqq & -\mathrm{R} \cos \theta \log \left|\mathrm{k}_{2} \mathrm{r}_{1} / \mathrm{ka}\right|+\pi \mathrm{R} \sin \theta \\
\leqq & -\mathrm{R}\left[\cos \alpha|\log | \mathrm{k}_{2} \mathrm{r}_{1} / \mathrm{ka} \mid-\pi \sin \alpha\right],-\alpha \leqq \theta \leqq \alpha,
\end{aligned}
$$

since $\log (\mathrm{r} / \mathrm{a})>0$. The expression in parenthesis is positive, since the quantity $r_{1}$ is chosen in accordance with the relation (4.5), so that the third 0-term in (3.16) is integrable on $\mathrm{W}_{\mathrm{o}}$.

It remains to prove that the integral present on the right hand side of (3.15) is equal to zero, and this may be carried out by closing the contour in the sector $-\alpha \leqq \theta \leqq \alpha$ by means of a suitable sequence of curves $C_{n}^{\prime}$ which avoid the zeros of sin $u \pi$ and which recede to infinity as $n \rightarrow \infty$. We may take $C_{n}^{\prime}$ to be the curve which comprises the part of the straight line $\operatorname{Re}(u)=n+\frac{1}{2}$ intercepted inside the sector $\mid$ argu $\mid \leqq \delta$ and which is connected to $w_{0}$ by means of circular arcs of suitable radius. The formulas (3.8), (3.10) can be applied to estimate the magnitude of the integrand on $C_{n}^{\prime}$ since $|\sin u \pi|=\cosh (s \pi) \geqq \frac{1}{2} \exp |s \pi|$ in the vicinity of the zeros whilst $|\sin u \pi| \sim \frac{1}{2} \exp |s \pi|$ elsewhere on $C_{n}^{\prime}$ we see that (3.16) is applicable so that the integral along these curves tends to zero as $\mathrm{n} \rightarrow \infty$. Hence the contour may be closed in the manner described whereupon it is seen that the integral is equal to zero since the integrand is analytic in the domain to the right of $W_{0} T^{T}$ is proves (3.15) and on taking the limit of each side of (3.14) as $c \rightarrow 0$ we obtain the equation (3.1) to be proved.

It is now possible to state the main theorem:

THEOREM 2. Let the function $f(r)$ be defined as in Theorem 1, then, if $r>a$,

$$
f(r)=-i \pi \lim _{c \rightarrow 0} \sum_{u_{n}} \frac{u J_{u}(k a) H_{u}^{(1)}(k r) F(u) e^{c u^{2}}}{(\partial / \partial u) H_{u}^{(1)}(k a)}
$$

in which the summation extends over all of those zeros $u_{n}$ of $H_{u}^{(1)}(k a)$, regarded as a function of the order $u$, that are positioned in the first quadrant of the complex u-plane.

The expansion appearing in the formula (3.17) follows on subtracting (3.1) from (2.1) which leads to the formula

$$
\begin{aligned}
f(r)=\frac{1}{2} \lim _{c \rightarrow 0} \int_{L} \frac{u\left[J_{u}(k r) H_{u}^{(1)}(k a)-J_{u}(k a) H_{u}^{(1)}(k r)\right] F(u) e^{c u^{2}} d u}{H_{u}^{(1)}(k a)} \\
-i \pi \lim _{c \rightarrow 0} \sum_{n} \frac{u_{u}(k a) H_{u}^{(1)}(k r) F(u) e^{c u^{2}}}{(\partial / \partial u) H_{u}^{(1)}(k a)}
\end{aligned}
$$

It is easily verified that the integrand present in the integral on the right hand of the preceding equation is an odd function of $u$, so that the value of the integral taken along the entire imaginary axis is zero. To see this property we appeal to the 
identity $\mathrm{H}_{-u}^{(1)}(\mathrm{kr})=\mathrm{e}^{\mathrm{iu \pi} \mathrm{H}_{\mathrm{u}}^{(1)}}(\mathrm{kr})$ which in conjunction with the definition of $\mathrm{F}(\mathrm{u})$ shows that $F(-u)=e^{i u \pi} F(u)$ so that the quotient $F(u) / H_{u}^{(1)}(k a)$ is an even function of $u$. Since it has already been proved, (2.7), that the cross product of Bessel functions appearing in (3.18) is an even function of $u$, it follows that the integrand in question reduces to an odd function of $u$. Hence the integral term present in (3.18) is actually equal to zero, so that this formula reduces to the expansion stated in the theorem.

4. In this section the various bounds needed in the paper are developed. A suitable bound on the Hankel function can be obtained from the formula, Magnus et al [9, p. 81]

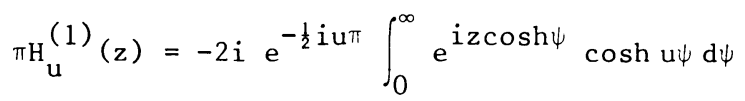

If we set $z=k r=\left(k_{1}+i k_{2}\right) r$ and $u=t+i s$ it is found, since $|\operatorname{coshu} \psi| \leqq \cosh t \psi$, that

$$
\pi\left|H_{u}^{(1)}(k r)\right| \leqq 2 e^{\frac{1}{2} s \pi} \int_{0}^{\infty} e^{-k_{2} r \cosh \psi} \cosh t \psi d \psi
$$

The integral appearing on the right hand side of this inequality is equal to the Bessel function $k_{t}\left(k_{2} r\right)$ so that,

$$
\pi\left|H_{u}^{(1)}(k r)\right| \leqq 2 e^{\frac{1}{2} s \pi} K_{t}\left(k_{2} r\right)
$$

The formula (2.4) follows from this result by setting $u=i s, t=0$. If we use the inequalities $2 \cosh \psi \geqq \exp \psi$ and $\cosh t \psi \leqq \exp \psi \psi$ in the integral in (4.1) and then introduce the variable $v=\exp \psi$, we find, if $t>0$, that

so that

$$
\left|\pi H_{u}^{(1)}(k r)\right| \leqq 2 e^{\frac{1}{2} s \pi} \int_{0}^{\infty} \exp \left(-\frac{1}{2} k_{2} r v\right) v^{t-1} d v
$$

$$
\left|\pi H_{u}^{(1)}(k r)\right| \leqq 2 e^{\frac{1}{2} s \pi} \Gamma(t)\left(2 / k_{2} r\right)^{t}
$$

With the aid of this result we may now proceed to deduce a bound on the transform $\mathrm{F}(\mathrm{u})$ - A bound adequate for the present purpose can be obtained by decomposing the domain of integration into the parts $\left(a, r_{1}\right),\left(r_{1}, \infty\right)$ so that

where

$$
F(u)=\int_{a}^{r} 1_{u} H_{u}^{(1)}(k r) f(r) \frac{d r}{r}+\int_{r_{1}}^{\infty} H_{u}^{(1)}(k r) f(r) \frac{d r}{r}
$$

$$
\mathrm{r}_{1}>\mathrm{r}\left|\mathrm{k} / \mathrm{k}_{2}\right|^{\pi \tan \alpha}
$$

When $u$ is large the integral over the bounded interval $\left(a, r_{1}\right)$ can be estimated by inserting the asymptotic expression (2.17) with $x$ replaced by $\mathrm{kr}$ therein. This leads to the estimate

$$
\begin{aligned}
& \int_{a}^{r} 1 H_{u}^{(1)}(k r) f(r) \frac{d r}{r}=\frac{\Gamma(u)}{i \pi} \int_{a}^{r} 1(k r / 2)^{-u} f(r) \frac{d r}{r}-\frac{e^{-i u \pi}}{i \Gamma(u+1) \sin u \pi} \int_{a}^{r} 1(k r / 2){ }^{u} f(r) \frac{d r}{r} \\
& =O\left[(k a / 2)^{-u} \Gamma(u)\right]+0\left[\frac{e^{-i u \pi}\left(k r_{1} / 2\right)^{u}}{\Gamma(u+1) \sin u \pi}\right]
\end{aligned}
$$

A bound on the $\left(r_{1}, \infty\right)$ integral in $(4.4)$ can be deduced from (4.3) which yields the inequality 


$$
\begin{aligned}
\int_{r_{1}}^{\infty} H_{u}^{(1)}(k r) f(r) \frac{d r}{r} & \leqq \frac{2}{\pi} e^{\frac{1}{2} s \pi} \Gamma(t) \int_{r_{1}}^{\infty}\left(k_{2} r / 2\right)^{-t}|f(r)| \frac{d r}{r} \\
& =0\left[e^{\frac{1}{2} s \pi} \Gamma(t)\left(k_{2} r_{1} / 2\right)^{-t}\right]
\end{aligned}
$$

On combining (4.6) and (4.7) it is found that

$$
F(u)=0\left[(k a / 2)^{-u} \Gamma(u)\right]+0\left[\frac{e^{-i u \pi}\left(k r_{1} / 2\right)^{u}}{\Gamma(u+1) \sin u \pi}\right]+0\left[e^{\frac{1}{2} s \pi} \Gamma(t)\left(k_{2} r_{1} / 2\right)^{-t}\right]
$$

This bound breaks down on the imaginary axis, since $t=0$ there. However an alternative bound valid in the vicinity of this axis can be obtained by applying the result (4.2). If we confine attention to some strip $0 \leqq t \leqq \lambda$, say, and recall that $k_{t}\left(k_{2} r\right)$ is an increasing function of $t$ but a decreasing function of $r$, we see from (4.2) that,

$$
\left|\pi H_{u}(k r)\right| \leqq 2 e^{\frac{1}{2} s \pi} K_{\lambda}\left(k_{2} r\right) \leqq 2 e^{\frac{1}{2} s \pi} K_{\lambda}\left(k_{2} a\right)
$$

and therefore that

$$
F(\text { is })=O\left[e^{\frac{1}{2} s \pi}\right]
$$

for $0 \leqq \operatorname{Re}(u) \leqq \lambda$.

It remains to establish the inequality (2.3), which can be deduced from the formula, Magnus et al [9, p. 79],

$$
\sqrt{\pi} \Gamma\left(\mathrm{u}+\frac{1}{2}\right) J_{u}(k r)=2(k r / 2)^{u} \int_{0}^{\frac{\pi}{2}} \cos (k r \cos \psi)(\sin \psi)^{2 u} d \psi
$$

On setting $u=i s$ and taking the modulus of each side we $f$ ind the equation

$$
\sqrt{\pi} \mid r\left(\text { is }+\frac{1}{2}\right) J_{\text {is }}(k r) \mid \leqq 2 e^{-s \beta} \int_{0}^{\frac{\pi}{2}} \cosh \left(k_{2} r \cos \psi\right) d \psi=\pi e^{-s \beta} I_{o}\left(k_{2} r\right)
$$

by Magnus et al [9]. The formula (2.3) follows from this on setting $\left|\Gamma\left(\frac{1}{2}+i s\right)\right|=$ $(\pi \operatorname{sechs} \pi)^{\frac{1}{2}} \geqq \sqrt{\pi} \exp \left(-\left|\frac{1}{2} s \pi\right|\right)$.

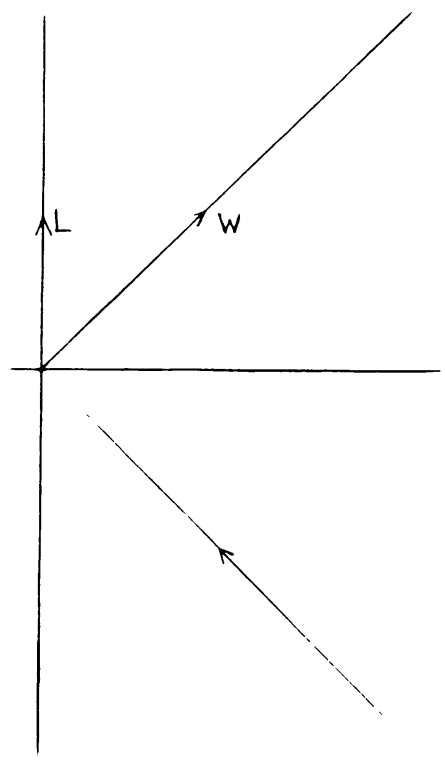




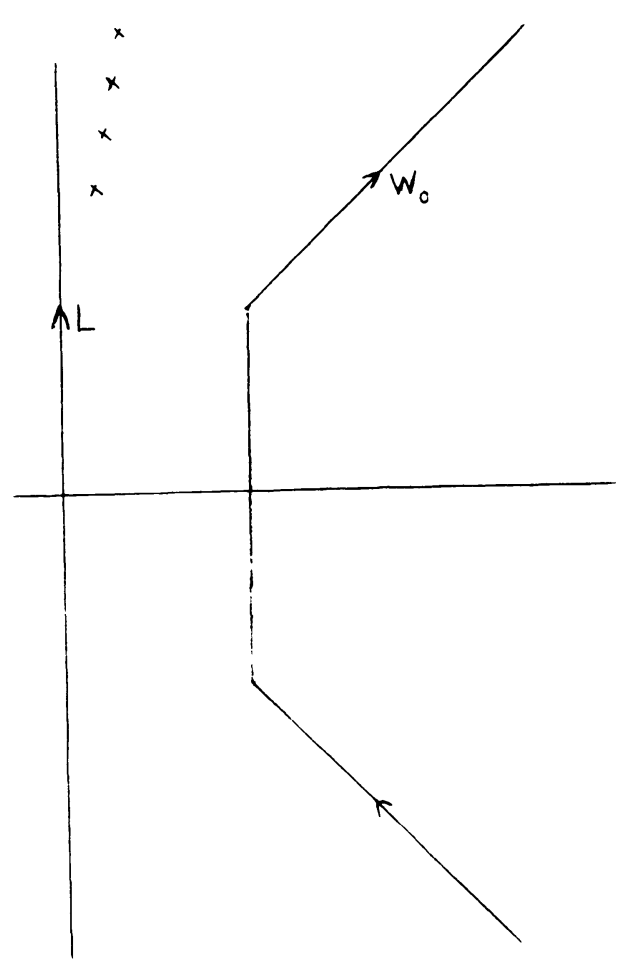

Fig. 2

\section{REFERENCES}

1. NAYLOR, D., CHOO, F.C. and BARCLAY, D.W. On an eigenfunction expansion associated with a condition of radiation, part 2, Proc. Cambridge Philos. Soc. 4 (1973)485-496.

2. WATSON, G.N. Theory of Bessel Functions, Second Edition, Cambridge University Press, London, 1958.

3. NAYLOR, D. and CHANG, P.H. On a formula of inversion, SIAM J. Math. Anal. 13 (1982) 1053-1071.

4. TITCHMARSH, E.C. Introduction to the Theory of Fourier Integrals, Oxford University Press, London, 1950.

5. COCHRAN, J.A. The zeros of Hankel functions as functions of their order, Numer. Math. $\underline{7}$ (1965) 238-250.

6. KELLER, J.B., RUBINOW, S.I. and GOLDSTEIN, M. Zeros of Hankel functions and poles of scattering amplitudes, J. Mathematical Physics 4 (1963) 829-832.

7. MAGNUS, W. and KOTIN, I. The zeros of the Hankel function as a function of its order, Numer. Math. 2 (1960) 228-244.

8. NAYLOR, D. On a non self adjoint eigenfunction ezpansion, SIAM J. Math. Anal. 9 (1978) 967-978.

9. MAGNUS, W., OBERHETTINGER, F. and SONI, R.P. Formulas and theorems for the Special Functions of Mathematical Physics, Springer-Verlag, New York, 1965. 


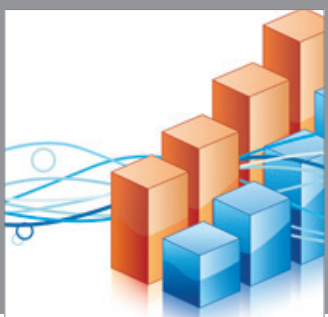

Advances in

Operations Research

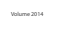

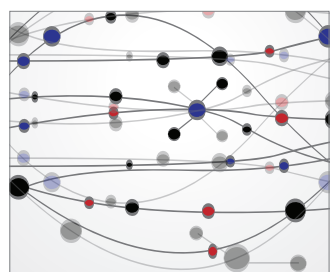

\section{The Scientific} World Journal
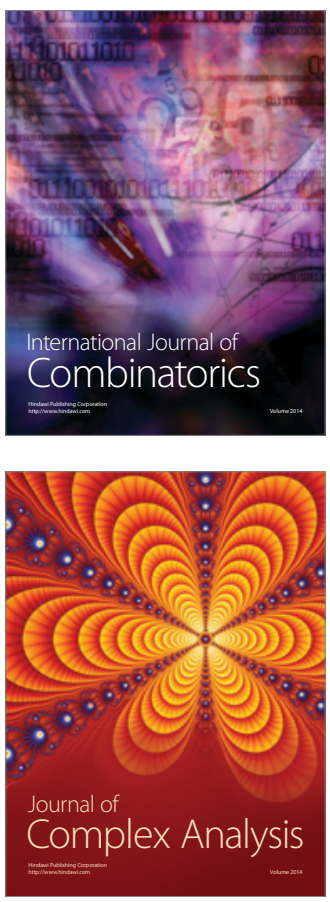

International Journal of

Mathematics and

Mathematical

Sciences
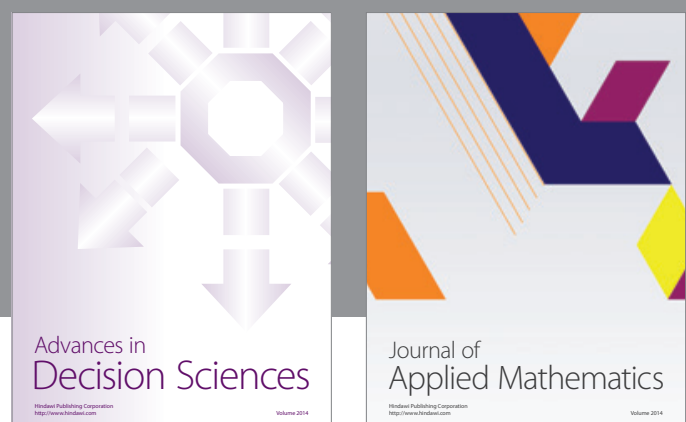

Journal of

Applied Mathematics
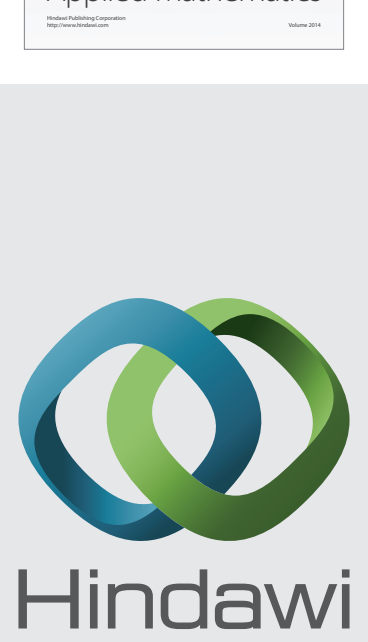

Submit your manuscripts at http://www.hindawi.com
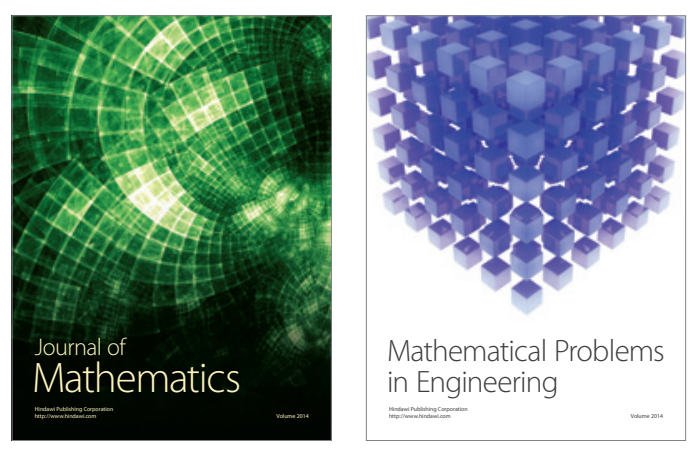

Mathematical Problems in Engineering
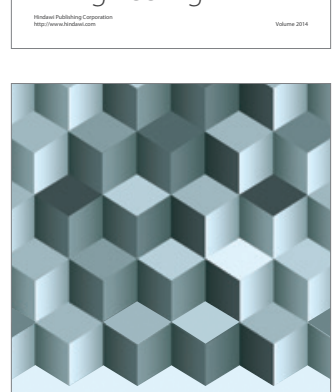

Journal of

Function Spaces
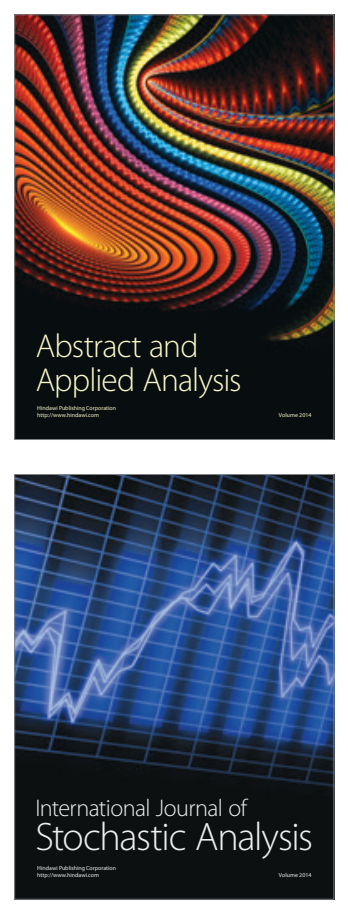

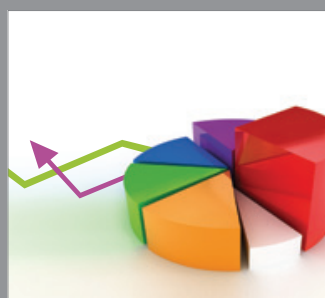

ournal of

Probability and Statistics

Promensencen
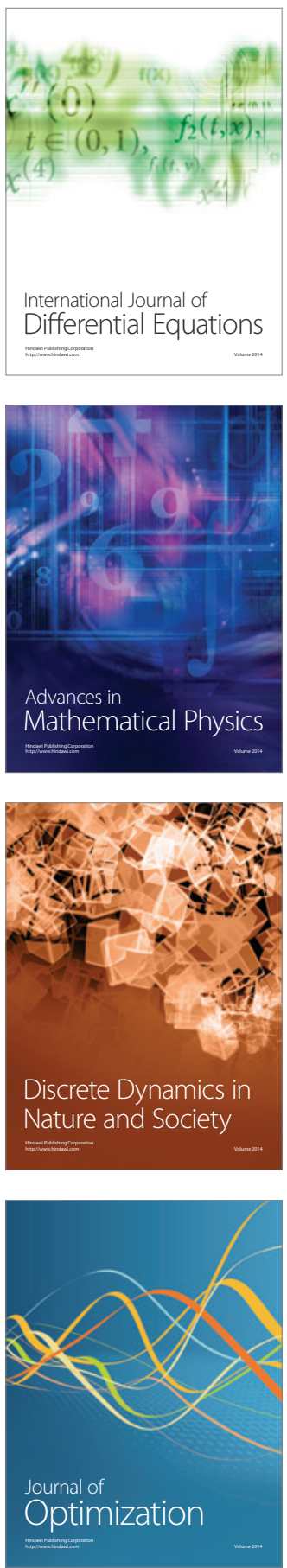